\title{
Numerical Simulation Research on Heat Charging Process of A New Type Thermal Energy Storage Equipment
}

\author{
Xiaolin Zhu ${ }^{1, a}$,Zhifeng Wang ${ }^{1, b}$ Shidong $\mathrm{Li}^{2, \mathrm{c}}$ \\ ${ }^{1}$ Key Laboratory of Solar Thermal Energy and Photovoltaic System, Institute of Electrical \\ Engineering, Chinese Academy of Sciences, Beijing, 100190, China \\ ${ }^{2}$ College of Mechanical and Electrical Engineering, Guangdong University of Petrochemical \\ Technology, Guangdong Maoming ,525000, China \\ azxl_good2005@163.com, ${ }^{b}$ zhifeng@163.com , ${ }^{c}$ lishidong0759@126.com
}

\begin{abstract}
Keywords: Thermal energy storage; PCM; Direct contact; Heat charge.
Abstract. In this paper, a 2D numerical simulation model was developed in FLUENT base on a new type thermal energy storage equipment. The results showed that the form of vertical pipes with nozzle holes can create fluid channels and a large number of vortices quickly, which improve the heat charging power, present the effective way to sweep the obstacles of transportation of thermal energy storage.
\end{abstract}

\section{Introduction}

In recent years, global energy and environmental issues become severe, the fossil energy resources are getting gradually exhausted. At the same time, a large number of solar energy, waste heat from industries and power stations, kept unused due to the long distance to the user sites[1]. Therefore, it is urgent to develop a efficient methods for transportation of low-grade and middle-grade heat over long distance. The traditional ways to transport thermal energy, which are normally based in sensible/latent heat of water, are limited within small range of heat storage capacity and heat charge power, a long time charging process decrease the amount of supplied heat ,take account of less heat loss and the best economic benefit[2], the transport distance is less than $10 \mathrm{~km}$ in general. The situation restrict application of thermal energy transport technology.

To solve the limit above, a phase change thermal energy storage technology with direct-contact heat exchanger had been studied [3,4], phase change material(PCM) blend with heat-transfer oil (HTO) in storage container for heat exchange, higher charge power of the technology is quite attractive, but due to HTO nozzle is easily blocked by solid PCM, circulation of thermal HTO is hindered, the heat charge power is low in initial stage of heat charge process[5], therefore, in this paper a new type thermal energy storage equipment was designed, base on the new design, numerical simulation study was carried out to acquire characteristics of flow, heat exchange and phase change in heat charge process, it is important to guide further optimization design for boosting charge power.

\section{Simulation methodology}

Modeling. A 2D-CFD model was built by FLUENT software to research the phase change behavior of the PCM in the new-type thermal energy storage equipment, base on the concept of direct contact heat exchange, which is HTO as heat transfer fluid to melt/cool PCM for heat storage or release. In the model, 20 nozzle holes are located at vertical pipes, diameter of the nozzle hole is $4 \mathrm{~mm}$, specific feature of structure and sizes as illustrated in Fig.1. The model has been simplified as follow: 1. PCM is homogeneous; 2.The phase change behavior in axial direction is identical; 3.The area under inlet pipe is ignored to avoid complicated mesh around inlet pipe. 4. Setting the velocity magnitude of thermal HTO normal to the inlet boundary (nozzle holes). 5. Wall of the model is adiabatic. The calculation domain divided into several zones for high quality mesh generating, the mesh include 3546 quadrangular elements for simulation, as illustrated in Fig.2. In the model, Enthalpy-porosity method 
was used for simulating phase change behavior of PCM, and VOF method was introduced to simulate the multiphase fluid[6].

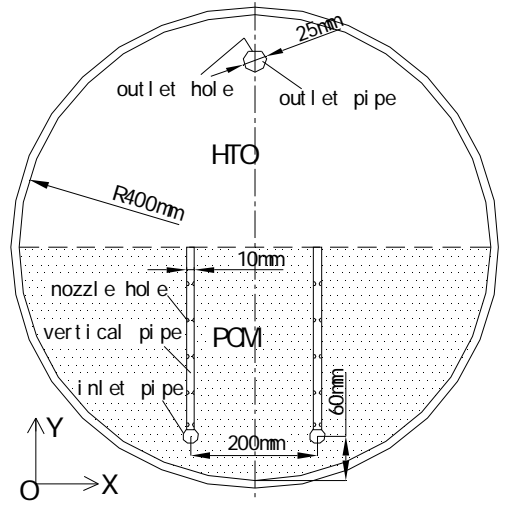

Fig.1. model of new-type thermal energy storage equipment

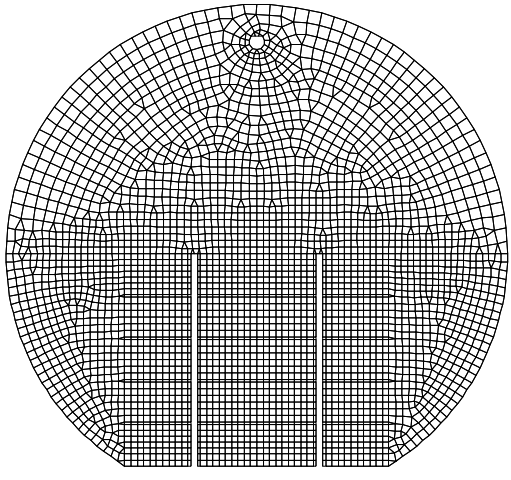

Fig.2. mesh

Equations. The following equations were introduced in model based on methods described above. Energy, mass and momentum conservation equation can express as Eq. (1), Eq. (2), Eq. (3).

$$
\frac{\partial(\rho h)}{\partial t}+\nabla(\rho v h)=\nabla(k \nabla t)+S_{e}
$$

Where $h$ is enthalpy, consists of sensible enthalpy $h_{s}$ and latent heat $\Delta h, \rho$ is density, $v$ is velocity of fluid, $t$ is time, $k$ is thermal conductivity, $S_{e}$ is energy source term.

$$
\frac{\partial(\rho v)}{\partial t}+\nabla(\rho v v)=\nabla(\mu \nabla v)-\nabla P+\rho g+S_{m}
$$

Where $\mu$ is dynamic viscosity, $P$ is pressure, $g$ is acceleration of gravity, $S_{m}$ is momentum source term.

$$
\frac{\partial \rho}{\partial t}+\nabla(\rho v)=0
$$

In Eq.(1), $\Delta h=\beta L, \beta$ is the liquid fraction, it could be calculated by Eq. (4),$L$ is the latent heat capacity of PCM.

$$
\beta=\left\{\begin{array}{c}
0, T<T_{s} \\
\frac{T-T_{s}}{T_{l}-T_{s}}, T_{s}<T<T_{l} \\
1, T>T_{l}
\end{array}\right.
$$

Where $T_{s}$ is solidus temperature, $T_{l}$ is liquidus temperature .

Boundary and initial conditions. The following boundary conditions were determined for solving equations above, PCM container wall set as adiabatic wall, initial flow velocity of HTO from every nozzle hole is $0.05 \mathrm{~m} / \mathrm{s}$, about equate $0.01 \mathrm{~kg} / \mathrm{s}$. Constant HTO temperature is $140^{\circ} \mathrm{C}, \rho=778 \mathrm{~kg} / \mathrm{m}^{3}, \mu$ is $0.003, k$ is $0.125 \mathrm{~W} / \mathrm{m} \cdot \mathrm{K}$. initial temperature of the model is $25^{\circ} \mathrm{C}$. Melting temperature of PCM is $118^{\circ} \mathrm{C}$, Thermo-physical properties of the PCM as illustrated in Tab 1.

Table 1. Thermo-physical properties of $\mathrm{PCM}^{[7,8]}$

\begin{tabular}{cccccc}
\hline$T\left({ }^{\circ} \mathrm{C}\right)$ & $\rho\left(\mathrm{kg} / \mathrm{m}^{3}\right)$ & $c(\mathrm{~J} / \mathrm{kg} \cdot \mathrm{K})$ & $\mu(\mathrm{kg} / \mathrm{m} \cdot \mathrm{s})$ & $k(\mathrm{~W} / \mathrm{m} \cdot \mathrm{K})$ & $L(\mathrm{~J} / \mathrm{kg})$ \\
\hline 20 & 1480 & 1350 & 0.02895 & 0.732 & 339000 \\
140 & 1300 & 2740 & 0.01602 & 0.326 & 339000 \\
\hline
\end{tabular}

\section{Results and discussion}

The simulation includes two stages: (I) fluid channel forming stage and (II) circulation stage. In the stage (I), the inlet flow rate is $0.01 \mathrm{~kg} / \mathrm{s}$, due to solid PCM blocking, thermal HTO from multiple nozzle holes injected into PCM continually, the PCM is compressed and heated by thermal HTO, then 
the thermal HTO raise along the thermal vertical pipes $\left(140^{\circ} \mathrm{C}\right)$. As shown in Fig. 3, when Time $=40 \mathrm{~s}$, narrow fluid channels around vertical pipes had been formed quickly, thermal HTO passed through the fluid channels to blend with cold HTO and flow out by outlet hole, larger flow velocity of thermal $\mathrm{HTO}(>0.2 \mathrm{~m} / \mathrm{s})$ only appeared on top area of the vertical pipe, in the stage (II), when Time $=1012 \mathrm{~s}$, a mass of cold HTO above PCM had been heated to $127^{\circ} \mathrm{C}(400 \mathrm{~K})$ by convective heat transfer, which is highter than melt temperature of PCM, therefore, diameter of the fluid channels expanded from top to bottom of PCM gradually, and the largest flow velocity increased to $0.38 \mathrm{~m} / \mathrm{s}$, it means that convective heat transfer strengthen melting rate of top PCM, profile of fluid channels are presented obviously. When Time $=3600$ s, it is observed that the temperature contour profile is a shape of "W", the temperature of fluid channels is higher than PCM, and maximum velocity increase in small.

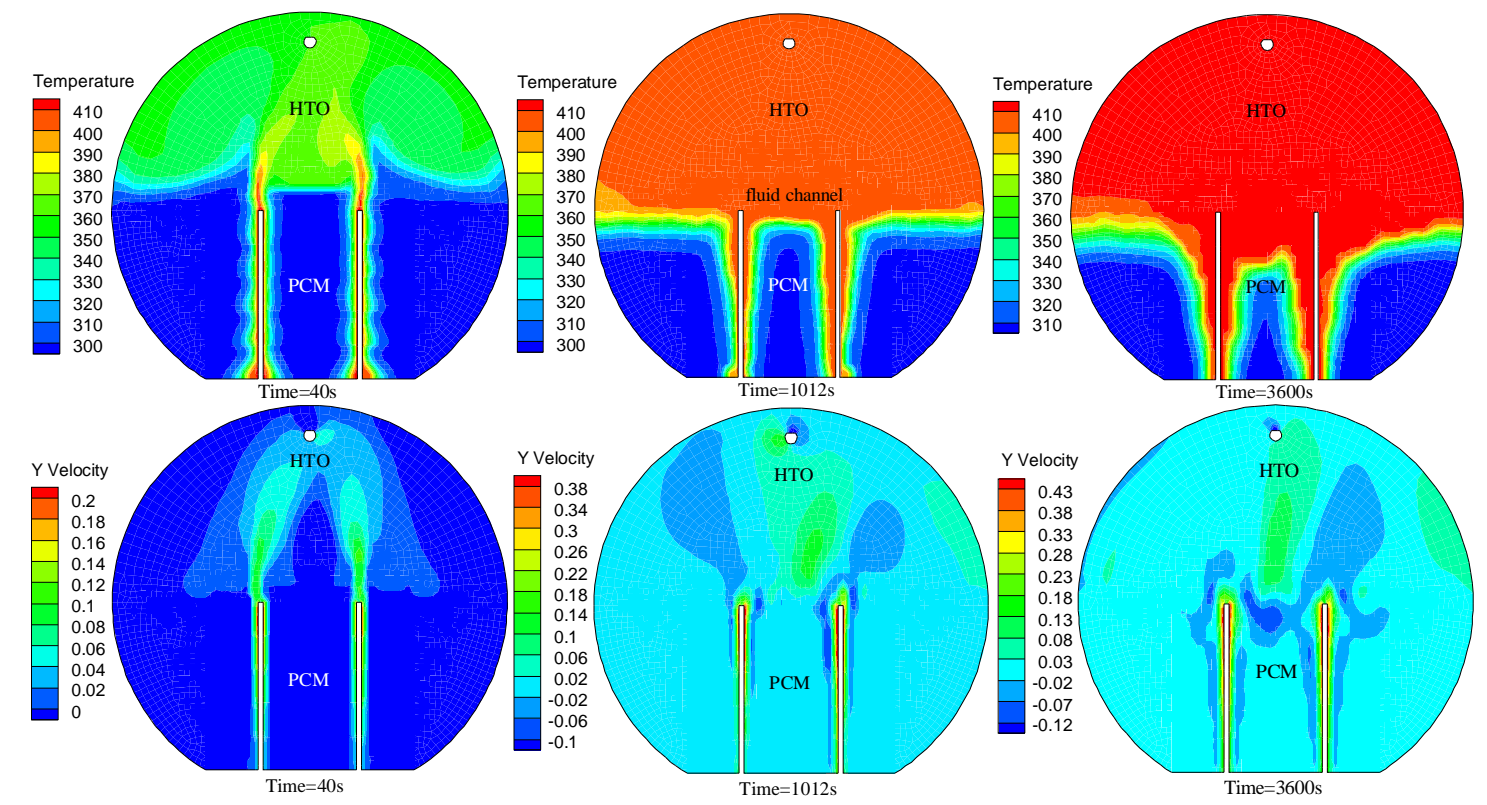

Fig.3. contour of temperature and velocity

This is because of density of liquid PCM is lower than solid PCM, and higher than density of thermal HTO, so it will locate at the top of the solid PCM along with the thermal HTO. Due to the force convection of the HTO and nature convection of the liquid PCM, the heat transfer between HTO and surface of solid PCM is reinforced, the PCM between fluid channels has a higher melting rate than the other parts, inside the solid PCM, heat conduction still dominates heat transfer. As shown in Fig. 4, the contour of density reveal layered situation of melting PCM, thermal HTO and solid PCM, By the vector of velocity, we know that due to difference of density, velocity and structure, small scale vortexes almost appear in melting PCM layer and large scale vortexes appear in HTO layer, which is main form of force convection and nature convection. The vortexes accelerate melting rate of PCM, it is obvious by contrasting liquid fraction of 3600s with 5442s. The area between two liquid channels will melt completely first, the corners of the equipment melt slowly, therefore, it is necessary to optimize the structure for promoting melting rate, so the additional vertical pipes will be set at the corners of the storage equipment, as shown in Fig. 4, this will helps to improve charging power.

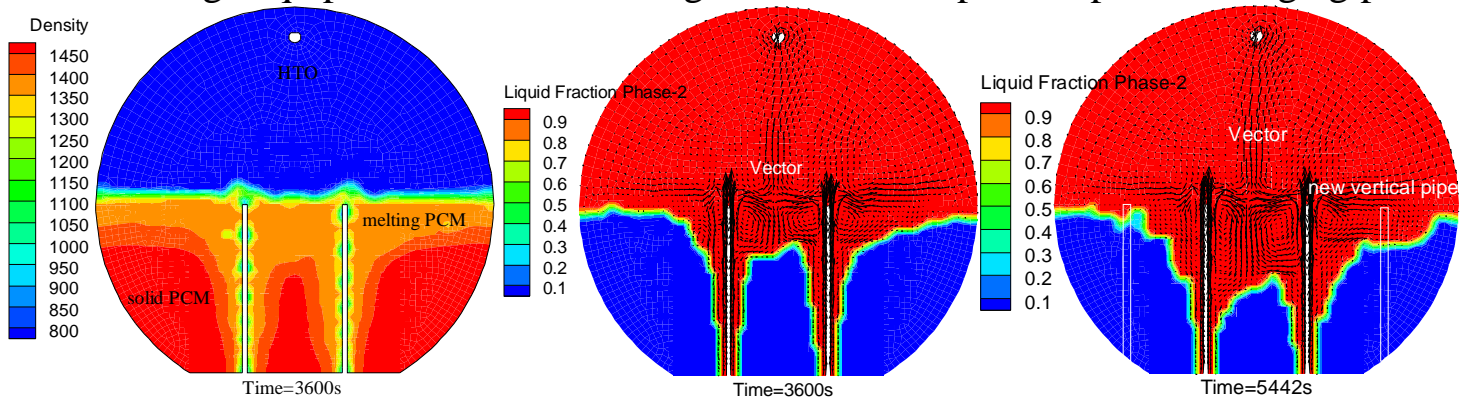

Fig.4. contour of density and vector of velocity and liquid fraction 


\section{Conclusions}

In the paper, a 2D numerical simulation model base on thermal energy storage equipment was developed for the simulation of charging process, the result show that the form of vertical pipes with nozzle holes can create fluid channels and a large number of vortices quickly, enhance intensity of nature and force convection, accelerate melting rate of PCM, improve the charging power, present the effective way to sweep obstacles of transportation of thermal energy storage.

\section{Acknowledgements}

This work was financially supported by the PhD Start-up Fund of Natural Science Foundation of Guangdong Province (2014A030310376); Guangdong Province Ordinary University Youth Innovative Talents Project (631051) and The PhD Start-up Fund of Natural Science Foundation of Guangdong Province (513090).

\section{References}

[1] Q. Ma, L.Luo and R.Z. Wang, A review on transportation of heat energy over long distance: Exploratory development, in Renewable and Sustainable Energy Reviews, Vol. 13 (2009), pp.1532-1540.

[2] Li HL, Wang WL, Yan J, Dahlquist E. Economic assessment of the mobilized thermal energy storage (M-TES) system for distributed heat supply ,in Appl Energy Vol.104 (2013),pp.78-86.

[3] Akihide Kaizawa, Hiroomi Kamano, Thermal and flow behaviors in heat transportation container using phase change material, in Energy Conversion and Management ,Vol.49 (2008) , p.698-706.

[4] Takahiro Nomura, Masakatsu Tsubota, Performance analysis of heat storage of direct-contact heat exchanger with phase-change material in Applied Thermal Engineering, Vol. 58(2013), p.108-113.

[5] Weilong Wang, Shiquan He.A combined experimental and simulation study on charging process of Erythritol-HTO direct-blending based energy storage system, in A combined experimental and simulation study on charging process of Erythritol-HTO direct-blending based energy storage system, Vol. 83 (2014), pp.306-313.

[6] FLUENT. Theory guide. In Chapters: "Modeling Solidification and Melting" and "Modeling Multiphase Flows",2009.

[7] Wang WL, Yan JY, Nyström J, Dahlquist E. Thermal performance of the mobilized thermal energy storage system. in International conference on applied energy, May 16-18, 2011, Perugia, Italy.

[8] Kaizawa A, Kamano H, Kawai A, et al. Thermal and flow behaviors in heat transportation container using phase change material in Energy Convers Manage. Vol. 49 (2008) , p.698-706. 